\title{
Large amplitude oscillations for a class of symmetric polynomial differential systems in $\mathbb{R}^{3}$
}

\author{
JAUME LLIBRE $^{1}$ and MARCELO MESSIAS ${ }^{2}$ \\ ${ }^{1}$ Departament de Matemàtiques, Universitat Autónoma de Barcelona, 08193 Bellaterra, Barcelona, Catalonia, Spain \\ ${ }^{2}$ Departamento de Matemática, Estatística e Computação, Faculdade de Ciências e Tecnologia da UNESP \\ Caixa Postal 467, 19060-900 Presidente Prudente, SP, Brazil
}

Manuscript received on January 17, 2007; accepted for publication on September 27, 2007;

presented by MANFREDO DO CARMO

\begin{abstract}
In this paper we study a class of symmetric polynomial differential systems in $\mathbb{R}^{3}$, which has a set of parallel invariant straight lines, forming degenerate heteroclinic cycles, which have their two singular endpoints at infinity. The global study near infinity is performed using the Poincaré compactification. We prove that for all $n \in \mathbb{N}$ there is $\varepsilon_{n}>0$ such that for $0<\varepsilon<\varepsilon_{n}$ the system has at least $n$ large amplitude periodic orbits bifurcating from the heteroclinic loop formed by the two invariant straight lines closest to the $x$-axis, one contained in the half-space $y>0$ and the other in $y<0$.
\end{abstract}

Key words: infinite heteroclinic loops, periodic orbits, symmetric systems.

\section{INTRODUCTION}

In this paper we study the following class of symmetric polynomial differential systems in $\mathbb{R}^{3}$

$$
\begin{aligned}
& \dot{x}=\frac{d x}{d t}=y, \\
& \dot{y}=\frac{d y}{d t}=z, \\
& \dot{z}=\frac{d z}{d t}=p(y)+\varepsilon q(x) z,
\end{aligned}
$$

where $\varepsilon$ is a small positive real parameter,

$$
p(y)=\sum_{i=0}^{m} a_{i} y^{i} \quad \text { and } \quad q(x)=\sum_{i=1}^{m / 2} b_{2 i-1} x^{2 i-1},
$$

AMS Classification: Primary: 58F21; Secondary: 34C05, 58F14.

Correspondence to: Marcelo Messias

E-mail: marcelo@fct.unesp.br 
with $m$ an even natural number, $a_{0}>0$ and $b_{m-1}>0$. Under these assumptions system (1) has no singular points in $\mathbb{R}^{3}$. This system can be extended to an analytic system on a closed ball of radius one, whose interior is diffeomorphic to $\mathbb{R}^{3}$ and its boundary (a 2-dimensional sphere $\mathbb{S}^{2}$ ) plays the role of infinity. The technique for making such an extension is called the Poincaré compactification, which is described in detail in Appendix 1.

We suppose that the polynomial $p(y)$ which appears in the third equation of system (1) has $k \geq 2$ simple real roots $r_{i}, i=1, \ldots, k$, with at least two of them having opposite signs. In this way the system has $k$ parallel invariant straight lines given by

$$
\gamma_{i}=\left\{\gamma_{i}(t)=(x(t), y(t), z(t))=\left(r_{i} t, r_{i}, 0\right) \in \mathbb{R}^{3}: t \in \mathbb{R}\right\}
$$

These invariant straight lines tend toward two diametrally opposite singular points at infinity when $t \rightarrow$ $\pm \infty$, corresponding to the endpoints of the $x$-axis, after the Poincaré compactification. In fact, each straight line $\gamma_{i}$ reaches the points at infinity with slope $r_{i}$ in a sense that we shall describe in the Subsection 2.2. Consider $r_{1}$ and $r_{2}$ the real roots of $p(y)$, with $r_{1}$ the largest negative and $r_{2}$ the smallest positive root. In this way, the invariant lines $\gamma_{1}=\left\{\left(r_{1} t, r_{1}, 0\right): t \in \mathbb{R}\right\}$ and $\left.\gamma_{2}=\left\{r_{2} t, r_{2}, 0\right): t \in \mathbb{R}\right\}$ together with the two singular points at infinity located at the end of the $x$-axis form a degenerate heteroclinic loop $L$.

It is important to observe that system (1) is invariant under the symmetry

$$
S:(x, y, z, t) \rightarrow(-x, y,-z,-t) .
$$

This means that if $\gamma(t)=(x(t), y(t), z(t))$ is a solution of the system, then

$$
S(\gamma(t))=(-x(-t), y(-t),-z(-t))
$$

is a solution too. So, due to the symmetry, if $\gamma$ has a point on the $y$-axis, then the orbits $\gamma$ and its symmetric orbit $S(\gamma)$ with respect to the $y$-axis coincide. Moreover if $\gamma$ has two points on the $y$-axis, then $\gamma(t)$ is a symmetric periodic orbit. Therefore a way to find periodic orbits is to look for orbits having two points on the $y$-axis. This technique will be used here to prove the existence of large amplitude periodic orbits bifurcating from the loop $L$ described above.

Let $\delta>0$ but small. We take an open segment $\Gamma=\left\{(0, y, 0): r_{1}<y<\delta+r_{1}\right\}$ (note that $r_{1}<0$ ) of the $y$-axis with its left endpoint $\left(0, r_{1}, 0\right)$ on the heteroclinic loop $L=\gamma_{1} \cup \gamma_{2}$ and we will follow its image under the flow of system (1) until its first intersection with the plane $x=0$ near the point $\left(0, r_{2}, 0\right)$ of $L$, see Figure 4. We denote by $\pi$ the Poincaré map going from $x=0$ near $\left(0, r_{1}, 0\right)$ to $x=0$ near the point $\left(0, r_{2}, 0\right)$. Then we shall prove that $\pi(\Gamma)$ is a spiral near the point $\left(0, r_{2}, 0\right)$ giving finitely many turns for every $\varepsilon>0$ sufficiently small. This number of turns tends to infinity as $\varepsilon \rightarrow 0$. The orbits through the intersection points of $\pi(\Gamma)$ with the $y$-axis are periodic because, by construction, they have two points on the $y$-axis. Using these ideas in Section 2 we shall prove the following result. As usual we denote by $\mathbb{N}$ the set of positive integers.

THEOREM 1. For all $n \in \mathbb{N}$ there is $\varepsilon_{n}>0$ such that system (1) for $\varepsilon \in\left(0, \varepsilon_{n}\right)$ has at least $n$ periodic orbits near the heteroclinic loop $L$. 
The idea that heteroclinic loops to infinity can create a set of large amplitude periodic orbits (and even chaotic ones) has already appeared in several papers, see for instance (Newell et al. 1988).

Llibre, MacKay and Rodríguez (Llibre et al. 2004, preprint) study system (1) for the case where considering $p(y)=1-y^{2}$ and $q(x)=x$. In this case the system is equivalent, by a change of coordinates and a reparametrization of time, to the differential equation

$$
y^{\prime \prime \prime}+y^{\prime \prime} y+\lambda\left(1-y^{\prime 2}\right)=0
$$

which is related to boundary layer theory in fluid mechanics where it is know as the Falkner-Skan equation (see Guyon et al. 1991) for a derivation of this equation. See also (Sparrow and Swinnerton-Dyer 1995, 2002) for analytical information on the existence of periodic and other types of orbits in the Falkner-Skan equation. In fact, for this system, there is a hyperbolic subshift near the infinite heteroclinic loop (Llibre et al. 2004, preprint). But, in this paper, we will restrict attention to finding large amplitude periodic orbits and understanding the geometrical mechanism which create them.

\section{PROOF OF THE THEOREM 1}

In this section we shall prove our main result. The proof is constructive and will be presented in the four next subsections. In order to fix the notation we write the polynomial differential system (1) in $\mathbb{R}^{3}$ in the form

$$
\begin{aligned}
& \dot{x}=P^{1}(x, y, z)=y, \\
& \dot{y}=P^{2}(x, y, z)=z, \\
& \dot{z}=P^{3}(x, y, z)=p(y)+\varepsilon q(x) z,
\end{aligned}
$$

where $p(y)$ and $q(x)$ are given in (2) and $\varepsilon>0$ is a small parameter. In what follows we denote by $X$ the vector field associated to this system.

\subsection{THE HETEROCLINIC LOOP $L$}

Let $\gamma_{1}(t)=\left(r_{1} t, r_{1}, 0\right)$ and $\gamma_{2}(t)=\left(r_{2} t, r_{2}, 0\right)$ be the two invariant straight lines of system (1), related to the largest negative and the smallest positive real root of $p(y), r_{1}$ and $r_{2}$, respectively. The endpoints of these two lines at infinity in the Poincare compactification are the origins $p_{1}=(0,0,0)$ and $p_{2}=(0,0,0)$ of the local charts $V_{1}$ and $U_{1}$, respectively. For more details see Appendix 1.

\subsection{The LOCAL FLOW AT THE SINGULAR POINT $p_{1}$ AT INFINITY}

Using the results stated in Appendix 1, we have that the expression of the Poincaré compactification $p(X)$ in the local chart $V_{1}$ is

$$
\begin{aligned}
& \dot{z_{1}}=z_{1}^{2} z_{3}^{m-1}-z_{2} z_{3}^{m-1}, \\
& \dot{z_{2}}=z_{1} z_{2} z_{3}^{m-1}-\bar{p}\left(z_{1}, z_{3}\right)-\varepsilon \frac{z_{2}}{z_{3}} \bar{q}\left(z_{3}\right), \\
& \dot{z_{3}}=z_{1} z_{3}^{m},
\end{aligned}
$$


where

$$
\bar{p}\left(z_{1}, z_{3}\right)=\sum_{i=0}^{m} a_{i} z_{1}^{i} z_{3}^{m-i} \quad \text { and } \quad \bar{q}\left(z_{3}\right)=\sum_{i=1}^{m / 2} b_{2 i-1} z_{3}^{m-(2 i-1)} .
$$

We want to study the local flow of this system around the singular point $p_{1}=(0,0,0)$. The eigenvalues of the linear part of this flow at $p_{1}=(0,0,0)$ are 0,0 and $-\varepsilon b_{m-1}$. As we are considering $b_{m-1}>0$, the singular point $p_{1}$ has a two dimensional central manifold and the flow outside this manifold tends exponentially to it because of the negative eigenvalue $-\varepsilon b_{m-1}$. Now we shall study the flow on this central manifold. For more details on central manifolds see (Carr 1981, Chow and Hale 1982).

PROPOSITION 2. The invariant straight lines $\gamma_{i}$ in a neighborhood of $p_{1}$ are contained in the central manifold of the singular point $p_{1}$ of system (4).

Proof. From Theorem 1 of (Carr 1981, page 4), we know that there exists a center manifold to $p_{1}$ given by $z_{2}=h\left(z_{1}, z_{3}\right)$ in a neighborhood of $p_{1}$, which satisfies the conditions

$$
h(0,0)=D h(0,0)=0 \quad \text { and } \quad \dot{z_{2}}=\frac{d}{d t} h\left(z_{1}, z_{3}\right)=\frac{\partial h}{\partial z_{1}} \dot{z_{1}}+\frac{\partial h}{\partial z_{3}} \dot{z_{3}}
$$

(for more details see (Carr 1981, page 5)). Moreover, the flow on this center manifold is governed by the 2-dimensional system

$$
\begin{aligned}
& \dot{z_{1}}=z_{1}^{2} z_{3}^{m-1}-z_{3}^{m-1} h\left(z_{1}, z_{3}\right), \\
& \dot{z_{3}}=z_{1} z_{3}^{m} .
\end{aligned}
$$

Note that the straight line $z_{3}=0$ is filled of singular points.

Considering conditions (6) and the derivatives given in system (4), the function $h$ must satisfy the equation

$$
\left(z_{1}^{2} z_{3}^{m-1}-h z_{3}^{m-1}\right) \frac{\partial h}{\partial z_{1}}+z_{1} z_{3}^{m} \frac{\partial h}{\partial z_{3}}-\left(z_{1} z_{3}^{m-1} h-\bar{p}-\varepsilon \frac{h}{z_{3}} \bar{q}\left(z_{3}\right)\right)=0,
$$

or, equivalently,

$$
z_{3}^{m-1}\left(z_{1}^{2} \frac{\partial h}{\partial z_{1}}-h \frac{\partial h}{\partial z_{1}}+z_{1} z_{3} \frac{\partial h}{\partial z_{3}}-h z_{1}\right)+\bar{p}+\varepsilon \frac{h}{z_{3}} \bar{q}\left(z_{3}\right)=0 .
$$

Expanding the function $h\left(z_{1}, z_{3}\right)$ in power series in a neighborhood of $p_{1}$, and substituting it in the previous equation we obtain

$$
h\left(z_{1}, z_{3}\right)=-\frac{1}{\varepsilon b_{m-1}} \bar{p}\left(z_{1}, z_{3}\right)+O_{m+1}\left(z_{1}, z_{3}\right),
$$

where $\bar{p}\left(z_{1}, z_{3}\right)$ is given in (5). Since system (1) has the invariant straight lines $x=r t, y=r, z=0$, where $r$ is a real root of the polynomial $p(y)$ given in (2), it follows that system (4) has the invariant straight lines $z_{1}=r z_{3}, z_{2}=0$ (observe that we take $x=1 / z_{3}, y=z_{1} / z_{3}$ and $z=z_{2} / z_{3}$ in the local chart $V_{1}$ in the compactification procedure, see Appendix 1). Therefore, system (7) has also the invariant straight lines $z_{1}=r z_{3}$. So for system (7) we have that

$$
\dot{z}_{1}-\left.r \dot{z}_{3}\right|_{z_{1}=r z_{3}}=-z_{3}^{m-1} h\left(r z_{3}, z_{3}\right)=0 .
$$

Consequently $h\left(r z_{3}, z_{3}\right)=0$. In short, the invariant straight lines $\gamma_{i}$ in a neighborhood of $p_{1}$ are contained in the central manifold of the singular point $p_{1}$ of system (4) and they reach this point with slope $r_{i}$. 
Recall that $r_{1}$ and $r_{2}$ denote the real roots of $p(y)$, with $r_{1}$ the largest negative and $r_{2}$ the smallest positive. Such a roots exist by assumptions. We denote by $\gamma_{1}$ and $\gamma_{2}$ the two invariant straight lines associated to these two roots, respectively.

Proposition 3. On the center manifold of the singular point $p_{1}$ of system (4) and in a neighborhood of $p_{1}$ restricted to $z_{3}<0$, there exists a hyperbolic sector having as separatrices the invariant straight lines $\gamma_{1}$ and $\gamma_{2}$ restricted to this neighborhood.

ProOF. Again we use the notations introduced in the proof of Proposition 2. We consider now the invariant straight lines $\gamma(t)=(r t, r, 0)$ with $r$ a real root of the polynomial $p(y)$.

Suppose that $r<0$, then on the straight line $\gamma$ and on the half-plane $x<0$; i.e. on the half-straight line $\gamma$ contained in $V_{1}$, considering the change of coordinates in the compactification process (see Appendix 1 for details) we have that $z_{3}=1 / x<0, z_{1}=y / x>0, \dot{z}_{1}<0$ (recall that on these invariant straight lines $\left.h\left(z_{1}, z_{3}\right)=0\right)$ and $\dot{z}_{3}>0$.

Similarly for the straight line $\gamma$ with $r>0$ contained in $V_{1}$, we have that $z_{3}=1 / x<0, z_{1}=y / x<0$, $\dot{z}_{1}<0$ and $\dot{z}_{3}<0$.

In short the flow on the straight lines $\gamma_{1}$ and $\gamma_{2}$ in a neighborhood of $p_{1}$ is as it is described in Figure 1.

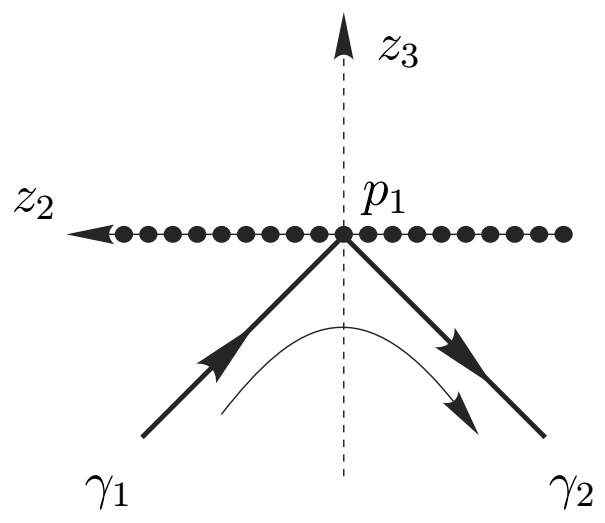

Fig. 1 - The flow on the center manifold.

Using (8) the differential system (7) on the central manifold $z_{2}=h\left(z_{1}, z_{3}\right)$ of the singular point $p_{1}$ for system (4) can be written, after a rescaling of the time by $z_{3}^{m-1}$, as

$$
\begin{aligned}
& \dot{z_{1}}=z_{1}^{2}+\frac{1}{\varepsilon b_{m-1}} \bar{p}\left(z_{1}, z_{3}\right)+O_{m+1}\left(z_{1}, z_{3}\right), \\
& \dot{z_{3}}=z_{1} z_{3} .
\end{aligned}
$$

We claim that the unique directions for tending to the origin of system (9) when the time $t \rightarrow \pm \infty$ are the ones given by the invariant straight lines $z_{1}=r z_{3}$ with $r$ a real root of the polynomial $p(y)$. Before proving the claim we end the proof of the proposition.

By Proposition 2 we know that the invariant straight lines $\gamma_{1}$ and $\gamma_{2}$ restricted to $V_{1}$ are solutions of system (9). Moreover from the previous paragraphs the solution defined by $\gamma_{1}$ ends at $p_{1}$, and the one 
defined by $\gamma_{2}$ starts at $p_{1}$. By the claim there are no other directions between the directions given by $\gamma_{1}$ and $\gamma_{2}$ for reaching the singular point $p_{1}$ in forward or backward time. Now from the differential system (7) on the central manifold $z_{2}=h\left(z_{1}, z_{3}\right)$ of the singular point $p_{1}$ and taking into account (8) we have that

$$
\left.\dot{z}_{1}\right|_{z_{1}=0}=\frac{a_{0}}{\varepsilon b_{m-1}} z_{3}^{2 m-1}+O\left(z_{3}^{2 m}\right) .
$$

On $z_{3}<0$ this expression is negative, so we have a hyperbolic sector. Recall that it is known that the local phase portraits of the singular point $p_{1}$ is a finite union of hyperbolic, elliptic and parabolic sectors (see, for instance, Andronov et al. 1973 or Dumortier et al. 2006).

Now we prove the claim. First we write system (9) in polar coordinates $(\rho, \theta)$ given by $z_{1}=\rho \cos \theta$ and $z_{3}=\rho \sin \theta$. The system becomes

$$
\begin{aligned}
& \dot{\rho}=\cos \theta\left[\rho^{2}+a \rho^{m} \bar{p}(\cos \theta, \sin \theta)\right]+O\left(\rho^{m+1}\right), \\
& \dot{\theta}=-a \sin \theta \bar{p}(\cos \theta, \sin \theta) \rho^{m-1}+O\left(\rho^{m}\right),
\end{aligned}
$$

where $a=a_{0} /\left(\varepsilon b_{m-1}\right)$. If a solution $(\rho(t), \theta(t))$ of this system tends to the origin when $t \rightarrow \pm \infty$ (i.e. $\rho(t) \rightarrow 0$ when $t \rightarrow \pm \infty)$, then the limit of $\theta(t)$ when $t \rightarrow \pm \infty$ exists, because the solution $(\rho(t), \theta(t))$ cannot spirals tending to the origin due to the existence of invariant straight lines through the origin.

Now from the differential system (10) it is clear that the unique directions $\theta^{*}$ in which a solution $(\rho(t), \theta(t))$ can reach the origin when $t \rightarrow \pm \infty$ are the zeros of $\sin \theta \bar{p}(\cos \theta, \sin \theta)$. That is, the directions of the invariant straight lines $z_{1}=r z_{3}$ with $r$ a real root of the polynomial $p(y)$, and $z_{3}=0$. Hence the claim is proved. Consequently Proposition 3 follows.

\subsection{Hamiltonian Structure associated to System (1) With $\varepsilon=0$}

In this Subsection we analyze the flow of system (1) for $\varepsilon=0$ in the $(y, z)$-plane. The equations for $\dot{y}$ and $\dot{z}$ of system (1) with $\varepsilon=0$ are the equations of the following Hamiltonian system with one degree of freedom

$$
\begin{aligned}
& \dot{y}=\frac{d y}{d t}=z, \\
& \dot{z}=\frac{d z}{d t}=p(y),
\end{aligned}
$$

with Hamiltonian given by

$$
H(y, z)=\frac{1}{2} z^{2}-\int p(y) d y=\frac{1}{2} z^{2}-\sum_{i=0}^{m} \frac{a_{i}}{(i+1)} y^{i+1} .
$$

Under the assumptions on the polynomial $p(y)$, this Hamiltonian system has $k \geq 2$ singular points, given by $\left(r_{i}, 0\right)$, where the $r_{i}$ 's are the real roots of $p(y)$. The jacobian matrix of system (11) calculated at one of these singular points is given by

$$
D X\left(r_{i}, 0\right)=\left(\begin{array}{cc}
0 & 1 \\
p^{\prime}\left(r_{i}\right) & 0
\end{array}\right)
$$

hence the singular point $\left(r_{i}, 0\right)$ is a saddle if $p^{\prime}\left(r_{i}\right)>0$, and a center if $p^{\prime}\left(r_{i}\right)<0$. 
Since $p\left(r_{1}\right)=0, r_{1}$ being the largest negative root of $p(y)$ and $p(0)=a_{0}>0$, it follows that $p^{\prime}\left(r_{1}\right) \geq 0$. We suppose that $p^{\prime}\left(r_{1}\right)>0$, and in a similar way we also assume that $p^{\prime}\left(r_{2}\right)<0$, where $r_{2}$ is the smallest positive root of $p(y)$. Therefore, $\left(r_{1}, 0\right)$ is a saddle and $\left(r_{2}, 0\right)$ is a center.

If all the real roots of $p(y)$ are simple, then the singular points $\left(r_{i}, 0\right)$ alternate between saddles and centers. In Figure 2, a possible phase portrait for the Hamiltonian system (11) is shown for the particular case in which the number of real roots of $p(y)$ is $k=4$ (see Example 4 below).

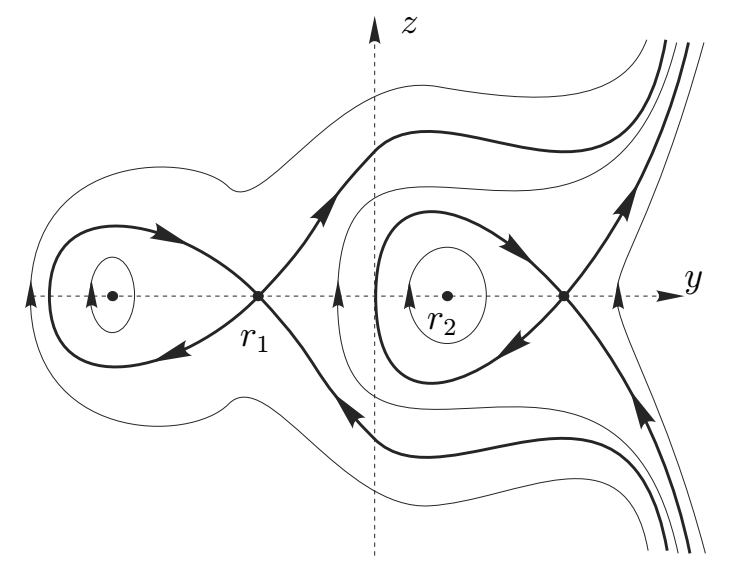

Fig. 2 - The orthogonal projection of the flow of system (1) with $\varepsilon=0$ into the $(y, z)$-plane.

From Figure 2 it is easy to understand the flow of system (1) when $\varepsilon=0$. The singular points of Figure 2 correspond to the invariant straight lines $\gamma_{i}$; i.e. the point $\left(r_{i}, 0\right)$ is the orthogonal projection with respect to the $x$-axis of $\gamma_{i}$ onto the $(y, z)$-plane. Observe that the invariant lines closest to the $z$-axis are $\gamma_{1}$ and $\gamma_{2}$.

We note that the flow of system (1), near the invariant straight line $\gamma_{2}$ when $\varepsilon=0$, is surrounded by invariant cylinders, the flow on these cylinders goes from $-\infty$ to $+\infty$ in the $x$ variable increasing monotonically because $\dot{x}=y>0$ in a neighborhood of $\gamma_{2}$. Hence, the flow of system (1) when $\varepsilon=0$ sufficiently near to $\gamma_{2}$ rotates around this straight line.

Let

$$
h_{2}=H\left(r_{2}, 0\right)=\sum_{i=0}^{m} \frac{a_{i}}{(i+1)} r_{2}^{i+1} .
$$

Then for the periodic orbits of the Hamiltonian system (11) surrounding the center $\left(r_{2}, 0\right)$ the Hamiltonian $H$ takes values in an open interval with endpoint $h_{2}$. Let $T(h)$ be the period of the periodic orbit of this center contained in $H=h$. We introduce the potential energy

$$
U(y)=-\int p(y) d y=-\sum_{i=0}^{m} \frac{a_{i}}{(i+1)} y^{i+1},
$$

associated to the Hamiltonian system (11). Then, from (Arnold 1980, page 20) we know that

$$
\lim _{h \rightarrow h_{2}} T(h)=\frac{2 \pi}{U^{\prime \prime}\left(r_{2}\right)}=\frac{2 \pi}{\beta},
$$

where

$$
\beta=-\sum_{i=1}^{m} i a_{i} r_{2}^{i-1} \text {. }
$$


Therefore the periods of the periodic orbits close to the point $\left(r_{2}, 0\right)$ are finite. This result will be used in the proof of Theorem 1 in the next Subsection.

EXAMPLE 4. If we take $p(y)=y^{4}-5 y^{2}+4$ and $q(x)=x+x^{3}$, then system (1) has the form

$$
\begin{aligned}
\dot{x} & =y, \\
\dot{y} & =z, \\
\dot{z} & =y^{4}-5 y^{2}+4+\varepsilon z\left(x+x^{3}\right) .
\end{aligned}
$$

The polynomial $p(y)$ has the four simple real roots: $-2,-1,1,2$. So this system has four invariant straight lines and for $\varepsilon=0$ the orthogonal projection with respect to the $x$-axis of its solutions on the $(y, z)$-plane is shown in Figure 2.

The expression of the Poincaré compactification of (13) in the local chart $V_{1}$ is

$$
\begin{aligned}
& \dot{z_{1}}=z_{1}^{2} z_{3}^{3}-z_{2} z_{3}^{3}, \\
& \dot{z_{2}}=z_{1} z_{2} z_{3}^{3}-\left(4 z_{3}^{4}-5 z_{1}^{2} z_{3}^{2}+z_{1}^{4}\right)-\varepsilon z_{2}\left(z_{3}^{2}+1\right), \\
& \dot{z_{3}}=z_{1} z_{3}^{3} .
\end{aligned}
$$

The origin $p_{1}=(0,0,0)$ is a singular point of this vector field with eigenvalues $0,0,-\varepsilon$, and then the system has a central manifold $z_{2}=h\left(z_{1}, z_{3}\right)$ and the flow of the system on this manifold is governed by the equation

$$
\begin{aligned}
& \dot{z_{1}}=z_{1}^{2}+\frac{1}{\varepsilon}\left(z_{1}^{4}-5 z_{1}^{2} z_{3}^{2}+4 z_{3}^{4}\right) \\
& \dot{z_{3}}=z_{1} z_{3},
\end{aligned}
$$

whose phase plane is as shown in Figure 3.

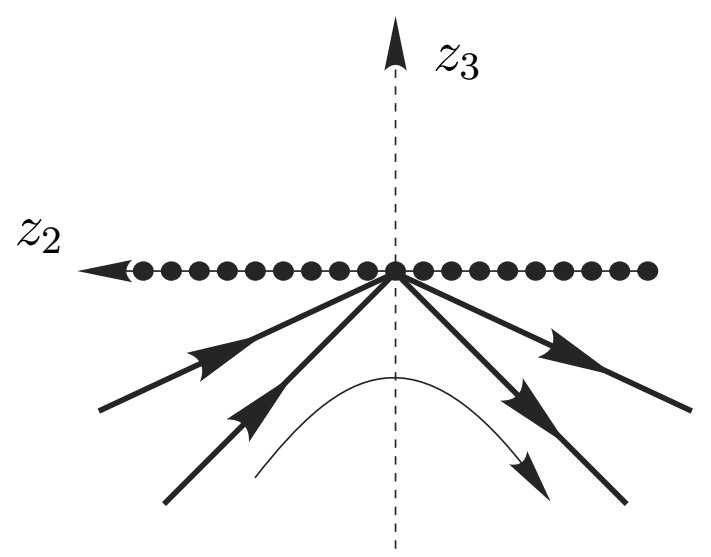

Fig. 3 - The local phase portrait on the center manifold for system (14).

\subsection{Construction of the Poincaré map And the proof of Theorem 1}

Let $\Sigma_{1}$ be a small square centered at the point $\left(0, r_{1}, 0\right)$ and contained in the plane $x=0$. Let $\Sigma_{2}$ be a small square centered at the point $\left(-k, r_{1}, 0\right)$ and contained in the plane $x=-k$ for $k>0$ sufficiently large. So, 
we can assume that $\Sigma_{2}$ is contained in a neighborhood of the point $p_{1}$ at infinity. Let $\Sigma_{3}$ be a small square centered at the point $\left(-k, r_{2}, 0\right)$ and contained in the plane $x=-k$. Hence, again we can suppose that $\Sigma_{3}$ is contained in a neighborhood of $p_{1}$. Finally, let $\Sigma_{4}$ be a small square centered at the point $\left(0, r_{2}, 0\right)$ and contained in the plane $x=0$.

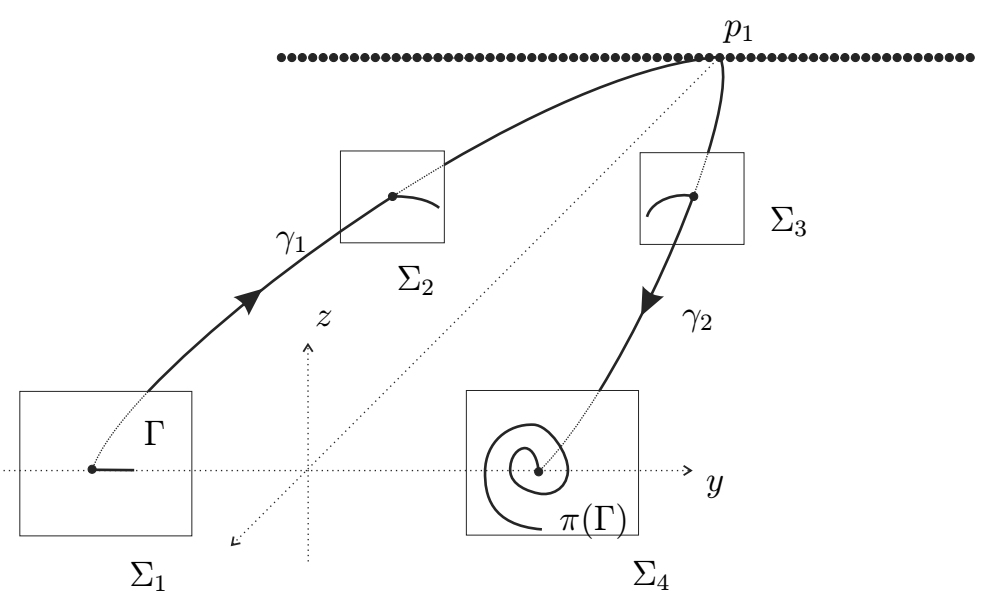

Fig. 4 - The Poincaré map.

We denote by $\pi$ the Poincaré map going from $\Sigma_{1}$ to $\Sigma_{4}$. Such a Poincaré map exists due to the existence of the heteroclinic loop $L$ and to the local phase portrait on the center manifold of $p_{1}$, see Proposition 3 . We split $\pi$ into three pieces. Let $\pi_{1}: \Sigma_{1} \rightarrow \Sigma_{2}, \pi_{2}: \Sigma_{2} \rightarrow \Sigma_{3}$ and $\pi_{3}: \Sigma_{3} \rightarrow \Sigma_{4}$. Therefore, $\pi=\pi_{3} \circ \pi_{2} \circ \pi_{1}$. See Figure 4.

Let $\delta>0$ but small. We consider the open segment $\Gamma=\left\{(0, y, 0): r_{1}<y<\delta+r_{1}\right\}$ on the $y$-axis having the left endpoint at $\left(0, r_{1}, 0\right)$. Then, since $\pi_{1}$ is a diffeomorphism (because the orbits going from $\Sigma_{1}$ to $\Sigma_{2}$ use only a bounded time), $\pi_{1}(\Gamma)$ is an arc in $\Sigma_{2}$ having the left endpoint at $\left(-k, r_{1}, 0\right)$.

We assume that $\Sigma_{2}$ and $\Sigma_{3}$ are in a neighborhood of $p_{1}$ where the local phase portrait studied in Subsection 2.2 holds. That is, $\gamma_{1}$ and $\gamma_{2}$ are in the center manifold of $p_{1}$ drawn in Figure 1, and the flow outside this center manifold tends exponentially to it. Therefore, by Proposition $3,\left(\pi_{2} \circ \pi_{1}\right)(\Gamma)$ is an arc in $\Sigma_{3}$ having the left endpoint at $\left(-k, r_{2}, 0\right)$.

Denote the time that the orbit $\gamma_{2}$ needs to go from the point $\left(-k, r_{2}, 0\right)$ to the point $\left(0, r_{2}, 0\right)$ by $\tau$. Then, if $\varepsilon>0$ is sufficiently small, by the theorem on continuous dependence on initial conditions and parameters, during a finite time the flow of system (1) is close to the flow of system (1) with $\varepsilon=0$, and in particular $\tau$ is close to $k$. So, during the time $\tau \approx k$ the orbits of system (1) near $\gamma_{2}$ passing through points of $\Sigma_{3}$ have made approximately $\beta k / 2 \pi$ turns (see expression (12) in Subsection 2.3). Consequently, $\left(\pi_{3} \circ \pi_{2} \circ \pi_{1}\right)(\Gamma)$ is an arc in $\Sigma_{4}$ which spirals to the point $\left(0, r_{2}, 0\right)$ giving approximately $\beta k / 2 \pi$ turns. Note that the number of turns tends to infinity when $k \rightarrow \infty$, and we can take $k$ as large as we want by taking the neighborhood of infinity where we choose $\Sigma_{2}$ and $\Sigma_{3}$ sufficiently small.

The orbits through the intersection points of $\pi(\Gamma)$ with the $y$-axis are periodic because, by construction, they have two points on the $y$-axis. This completes the proof of Theorem 1 .

A more complete analysis would produce a whole subshift passing near infinity, containing the derived 
symmetric periodic orbits. But in this note we are only interested in describing the geometrical mechanism which creates these large amplitude periodic orbits near the invariant straight lines $\gamma_{1}$ and $\gamma_{2}$.

\section{APPENDIX 1: POINCARÉ COMPACTIFICATION IN $\mathbb{R}^{3}$}

In $\mathbb{R}^{3}$ we consider the polynomial differential system

$$
\begin{aligned}
\dot{x} & =P^{1}(x, y, z), \\
\dot{y} & =P^{2}(x, y, z), \\
\dot{z} & =P^{3}(x, y, z),
\end{aligned}
$$

or equivalently its associated polynomial vector field $X=\left(P^{1}, P^{2}, P^{3}\right)$. The degree $n$ of $X$ is defined as $n=\max \left\{\operatorname{deg}\left(P^{i}\right): i=1,2,3\right\}$.

Let $\mathbb{S}^{3}=\left\{y=\left(y_{1}, y_{2}, y_{3}, y_{4}\right) \in \mathbb{R}^{4}:\|y\|=1\right\}$ be the unit sphere in $\mathbb{R}^{4}$, and

$$
\mathbb{S}_{+}=\left\{y \in \mathbb{S}^{3}: y_{4}>0\right\} \quad \text { and } \quad \mathbb{S}_{-}=\left\{y \in \mathbb{S}^{3}: y_{4}<0\right\}
$$

be the northern and southern hemispheres, respectively. The tangent space to $\mathbb{S}^{3}$ at the point $y$ is denoted by $T_{y} \mathbb{S}^{3}$. Then, the tangent plane

$$
T_{(0,0,0,1)} \mathbb{S}^{3}=\left\{\left(x_{1}, x_{2}, x_{3}, 1\right) \in \mathbb{R}^{4}:\left(x_{1}, x_{2}, x_{3}\right) \in \mathbb{R}^{3}\right\}
$$

is identified with $\mathbb{R}^{3}$.

We consider the central projections

$$
f_{+}: \mathbb{R}^{3}=T_{(0,0,0,1)} \mathbb{S}^{3} S_{+} \quad \text { and } \quad f_{-}: \mathbb{R}^{3}=T_{(0,0,0,1)} \mathbb{S}^{3} S_{-},
$$

defined by

$$
f_{+}(x)=\frac{1}{\Delta x}\left(x_{1}, x_{2}, x_{3}, 1\right) \text { and } f_{-}(x)=-\frac{1}{\Delta x}\left(x_{1}, x_{2}, x_{3}, 1\right),
$$

where

$$
\Delta x=\left(1+\sum_{i=1}^{3} x_{i}^{2}\right)^{1 / 2} .
$$

Through these central projections, $\mathbb{R}^{3}$ can also be identified with the northern and southern hemispheres. The equator of $\mathbb{S}^{3}$ is $\mathbb{S}^{2}=\left\{y \in \mathbb{S}^{3}: y_{4}=0\right\}$. Clearly, $\mathbb{S}^{2}$ can be identified with the infinity of $\mathbb{R}^{3}$.

The maps $f_{+}$and $f_{-}$define two copies of $X$, one $D f_{+} \circ X$ in the northern hemisphere and the other $D f_{-} \circ X$ in the southern one. Denote by $\bar{X}$ the vector field on $\mathbb{S}^{3} \backslash \mathbb{S}^{2}=\mathbb{S}_{+} \cup \mathbb{S}_{-}$which restricted to $\mathbb{S}_{+}$ coincides with $D f_{+} \circ X$ and restricted to $\mathbb{S}_{-}$coincides with $D f_{-} \circ X$.

In what follows we shall work with the orthogonal projection of the closed northern hemisphere to $y_{4}=0$. Note that this projection is a closed ball $B$ of radius one, whose interior is diffeomorphic to $\mathbb{R}^{3}$ and whose boundary $\mathbb{S}^{2}$ corresponds to the infinity of $\mathbb{R}^{3}$. We shall extend analytically the polynomial vector field $\bar{X}$ to the boundary, in such a way that the flow on the boundary is invariant. This new vector field on $B$ will be called the Poincaré compactification of $X$, and $B$ will be called the Poincaré ball. Poincaré 
introduced this compactification for polynomial vector fields in $\mathbb{R}^{2}$, and its extension to $\mathbb{R}^{m}$ can be found in (Cima and Llibre 1990).

The expression for $\bar{X}(y)$ on $\mathbb{S}_{+} \cup \mathbb{S}_{-}$is

$$
\bar{X}(y)=y_{4}\left(\begin{array}{lll}
1-y_{1}^{2} & -y_{2} y_{1} & -y_{3} y_{1} \\
-y_{1} y_{2} & 1-y_{2}^{2} & -y_{3} y_{2} \\
-y_{1} y_{3} & -y_{2} y_{3} & 1-y_{3}^{2} \\
-y_{1} y_{4} & -y_{2} y_{4} & -y_{3} y_{4}
\end{array}\right)\left(\begin{array}{l}
P^{1} \\
P^{2} \\
P^{3}
\end{array}\right),
$$

where $P^{i}=P^{i}\left(y_{1} /\left|y_{4}\right|, y_{2} /\left|y_{4}\right|, y_{3} /\left|y_{4}\right|\right)$. Written in this way $\bar{X}(y)$ is a vector field in $\mathbb{R}^{4}$ tangent to the sphere $\mathbb{S}^{3}$.

Now we can extend analytically the vector field $\bar{X}(y)$ to the whole sphere $\mathbb{S}^{3}$ by

$$
p(X)(y)=y_{4}^{n-1} \bar{X}(y)
$$

this extended vector field $p(X)$ is called the Poincaré compactification of $X$.

As $\mathbb{S}^{3}$ is a differentiable manifold, to compute the expression for $p(X)$ we can consider the eight local charts $\left(U_{i}, F_{i}\right),\left(V_{i}, G_{i}\right)$ where $U_{i}=\left\{y \in \mathbb{S}^{3}: y_{i}>0\right\}$, and $V_{i}=\left\{y \in \mathbb{S}^{3}: y_{i}<0\right\}$ for $i=1,2,3,4$; the diffeomorphisms $F_{i}: U_{i} \rightarrow \mathbb{R}^{3}$ and $G_{i}: V_{i} \rightarrow \mathbb{R}^{3}$ for $i=1,2,3,4$ are the inverses of the central projections from the origin to the tangent planes at the points $( \pm 1,0,0,0),(0, \pm 1,0,0),(0,0, \pm 1,0)$ and $(0,0,0, \pm 1)$, respectively. We now do the computations on $U_{1}$. Suppose that the origin $(0,0,0,0)$, the point $\left(y_{1}, y_{2}, y_{3}, y_{4}\right) \in \mathbb{S}^{3}$ and the point $\left(1, z_{1}, z_{2}, z_{3}\right)$ in the tangent plane to $\mathbb{S}^{3}$ at $(1,0,0,0)$ are collinear, then we have

$$
\frac{1}{y_{1}}=\frac{z_{1}}{y_{2}}=\frac{z_{2}}{y_{3}}=\frac{z_{3}}{y_{4}}
$$

and consequently

$$
F_{1}(y)=\left(\frac{y_{2}}{y_{1}}, \frac{y_{3}}{y_{1}}, \frac{y_{4}}{y_{1}}\right)=\left(z_{1}, z_{2}, z_{3}\right)
$$

defines the coordinates on $U_{1}$.

As

$$
D F_{1}(y)=\left(\begin{array}{cccc}
-y_{2} / y_{1}^{2} & 1 / y_{1} & 0 & 0 \\
-y_{3} / y_{1}^{2} & 0 & 1 / y_{1} & 0 \\
-y_{4} / y_{1}^{2} & 0 & 0 & 1 / y_{1}
\end{array}\right) \quad \text { and } \quad y_{4}^{n-1}=\left(\frac{z_{3}}{\Delta z}\right)^{n-1}
$$

the analytical field $p(X)$ becomes

$$
\frac{z_{3}^{n}}{(\Delta z)^{n-1}}\left(-z_{1} P^{1}+P^{2},-z_{2} P^{1}+P^{3},-z_{3} P^{1}\right)
$$

where $P^{i}=P^{i}\left(1 / z_{3}, z_{1} / z_{3}, z_{2} / z_{3}\right)$.

In a similar way we can deduce the expressions of $p(X)$ in $U_{2}$ and $U_{3}$. These are

$$
\frac{z_{3}^{n}}{(\Delta z)^{n-1}}\left(-z_{1} P^{2}+P^{1},-z_{2} P^{2}+P^{3},-z_{3} P^{2}\right),
$$


where $P^{i}=P^{i}\left(z_{1} / z_{3}, 1 / z_{3}, z_{2} / z_{3}\right)$ in $U_{2}$, and

$$
\frac{z_{3}^{n}}{(\Delta z)^{n-1}}\left(-z_{1} P^{3}+P^{1},-z_{2} P^{3}+P^{2},-z_{3} P^{3}\right),
$$

where $P^{i}=P^{i}\left(z_{1} / z_{3}, z_{2} / z_{3}, 1 / z_{3}\right)$ in $U_{3}$.

The expression for $p(X)$ in $U_{4}$ is $z_{3}^{n+1}\left(P^{1}, P^{2}, P^{3}\right)$ where $P^{i}=P^{i}\left(z_{1}, z_{2}, z_{3}\right)$. The expression for $p(X)$ in the local chart $V_{i}$ is the same as in $U_{i}$ multiplied by $(-1)^{n-1}$.

When we shall work with the expression of the compactified vector field $p(X)$ in the local charts we shall omit the factor $1 /(\Delta z)^{n-1}$. We can do that through a rescaling of the time.

We remark that all the points on the sphere at infinity in the coordinates of any local chart have $z_{3}=0$.

\section{ACKNOWLEDGMENTS}

The first author is partially supported by a MCYT/FEDER grant number MTM2005-06098-C02-01 and by a CICYT grant number 2005SGR 00550. Both authors are partially supported by the joint project CAPES-MECD grant HBP2003-0017.

The authors thank the referees for their suggestions which allowed them to improve the presentation of this paper.

\section{RESUMO}

Neste trabalho estudamos uma classe de campos vetoriais polinomiais com simetria, definidos no $\mathbb{R}^{3}$ e dependendo de um parâmetro real $\varepsilon$, que possui um conjunto de retas invariantes paralelas que tendem para dois pontos singulares no infinito, formando ciclos heteroclínicos degenerados. A análise global na vizinhança dos pontos no infinito é desenvolvida utilizando-se a compactificação de Poincaré. Provamos que para todo $n \in \mathbb{N}$ existe $\varepsilon_{n}>0$ tal que, para todo $0<\varepsilon<\varepsilon_{n}$, o sistema considerado possui pelo menos $n$ órbitas periódicas de grande amplitude, que bifurcam do ciclo heteroclínico formado pelas duas retas invariantes mais próximas do eixo- $x$, uma contida no semi-espaço $y>0$ e a outra contida no semi-espaço $y<0$.

Palavras-chave: ciclo heteroclínico infinito, órbitas periódicas, sistemas reversíveis.

\section{REFERENCES}

Andronov AA, Leontovich EA, Gordon II And Maier AL. 1973. Qualitative Theory of Second-Order Dynamical Systems. J Wiley \& Sons, New York.

ARNOLD VI. 1980. Geometrical Methods in the Theory of Ordinary Differential Equations. Graduate Texts in Mathematics. Springer-Verlag, New York, 60.

CARR J. 1981. Applications of centre manifold theory. Applied Math Sciencies, Springer-Verlag, New York, 35.

ChOw SN AND HALE JK. 1982. Methods of bifurcation theory. Grundlehren der Mathematischen Wissenschaften (Fundamental Principles of Mathematical Science). Springer-Verlag, New York, 251.

Cima A And Llibre J. 1990. Bounded polynomial vector fields. Trans Amer Math Soc 318: 557-579.

Dumortier F, Llibre J AND ARTÉs JC. 2006. Qualitative theory of planar differential systems. Universitext, Springer, New York. 
GuYOn E, Hulin JP AND PETIT L. 1991. Hydrodynamique physique. InterEditions/Editions du CNRS.

Llibre J, MacKay RS and Rodríguez G. 2004. Periodic Orbits Passing Near Infinity (preprint).

NeWEll AC, RAND DA And Russell D. 1988. Turbulent transport and the random ocurrence of coherent events. Phys D 33: 281-303.

Sparrow C AND Swinnerton-Dyer HPF. 1995. The Falkner-Skan equation I. The creation of strange invariant sets. J Differential Equations 119: 336-394.

Sparrow C AND SWInNERTON-Dyer HPF. 2002. The Falkner-Skan equation II. Dynamics and the bifurcations of $P$ - and $Q$-orbits. J Differential Equations 183: 1-55. 